\title{
Epigenetic modulation of the muscarinic type 3 receptor in salivary epithelial cells
}

\author{
Yong-Hwan Shin ${ }^{1}$, Meihong Jin ${ }^{1}$, Sung-Min Hwang ${ }^{1}$, Seul-Ki Choi ${ }^{1}$, Eun Namkoong ${ }^{1}$, Minkyoung Kim ${ }^{1}$, Moon-Yong \\ Park', Se-Young Choi ${ }^{1}$, Jong-Ho Lee ${ }^{2}$ and Kyungpyo Park ${ }^{1}$
}

Muscarinic receptors, particularly the type 3 subtype (M3R), have an important role in exocrine secretion. M3R normally function in HSG cells originated from human submandibular gland ducts, but not in A253 and SGT cells, derived from human submandibular carcinoma and salivary gland adenocarcinoma. However, the underlying mechanism of this suppression has remained elusive. In this study, we examined whether M3R function is suppressed by epigenetic modulation of the receptor. To this end, we investigated the mRNA transcript and protein levels of the M3R using reverse transcriptase-PCR, western blot, and confocal microscopy analyses. Global DNA methylation assays, methylation-specific $P C R$, and bisulfite sequencing were also performed to understand the epigenetic status of the M3R CpG island. We found that A253 cells expressed all subtypes of muscarinic receptors, except M3R, on the mRNA level. However, treatment of cells with 5-aza-2'-deoxycytidine (5-Aza-CdR), a DNA-demethylating agent, increased the expression levels of both M3R mRNA transcript and protein in proportion to the incubation period. 5-Aza-CdR completely restored the carbacholinduced calcium response, which was not observed in untreated A253 cells. In untreated A253 cells, all CG pairs from the 1st to 14th were methylated and 5-Aza-CdR treatment demethylated one of the methylated CG pairs. We also examined the methylation pattern of M3R CpG island in human cancer tissue. Interestingly, the result was very similar to those of A253 cells. All CG pairs in M3R CpG island were also methylated. Another salivary gland tumor cell line, SGT, also showed the similar methylation pattern, heavy methylation in M3R CpG island. It is concluded that CpG island in M3R is hypermethylated in cancer cell lines and human cancer. Our results further suggest that 5-Aza-CdR could potentially be used to restore the function of M3R, suppressed in some cancer cell types.

Laboratory Investigation (2015) 95, 237-245; doi:10.1038/labinvest.2014.150; published online 8 December 2014

Epigenetic regulation by $\mathrm{CpG}$ methylation has an important role in tumorigenesis, and is also crucial to mounting a successful response to cancer therapy. CpG islands in DNA are methylated de novo in a tissue-specific manner, and the patterns of methylation are fixed in subsequent cell divisions by methyltransferase activity. ${ }^{1}$ Recent studies have indicated that hypermethylation of $\mathrm{CpG}$ islands within the promoter and $5^{\prime}$ regions of genes is an important epigenetic mechanism for suppressing gene expression. ${ }^{2-4}$ DNA hypermethylation may directly affect the basal transcriptional machinery by altering the secondary structure of DNA and inducing chromosome remodeling through methyl group-binding proteins and histone deacetylase, thereby leading to transcriptional repression. ${ }^{5}$ 5-Aza-2'-deoxycytidine (5-Aza-CdR, decitabine) is a prodrug that has been shown to be phosphorylated, and thus activated, by deoxytidine kinase. 5-Aza-CdR is a nucleotide analog that is incorporated into DNA, where it irreversibly inactivates DNA methyltransferase (DNMT); 5Aza-CdR is also an S-phase-specific agent. ${ }^{6}$ The cytosine DNMT genes have a critical role in the establishment of the transcriptionally repressive complex. They function as de novo methylases to affect the methylation status of normally unmethylated CpG sites. ${ }^{7}$ The demethylation of DNA by 5Aza-CdR has also been shown to reactivate many genes that have been inactivated by hypermethylation. ${ }^{8-12}$ Thus, reduction in DNMT activities that likely block epigenetically mediated gene silencing might provide a novel clinical strategy to help prevent the leading cause of cancer death. ${ }^{13}$

Muscarinic cholinergic receptors are members of the heterotrimeric G-protein-coupled receptor (GPCR) superfamily.

\footnotetext{
${ }^{1}$ Department of Physiology, School of Dentistry, Seoul National University and Dental Research Institute, Seoul, Korea and ${ }^{2}$ Oral and Maxillofacial Surgery, School of Dentistry, Seoul National University and Dental Research Institute, Seoul, Korea

Correspondence: Professor K Park, Department of Physiology, School of Dentistry, Seoul National University and Dental Research Institute, 28 Yeongeondong, Chongnoku, Seoul 110-749, Republic of Korea.

E-mail: kppark@snu.ac.kr

Received 14 May 2014; revised 30 September 2014; accepted 15 October 2014
} 
Muscarinic cholinergic receptors are abundant in the central nervous system, as well as in non-neural tissues that are innervated by the parasympathetic nervous system. There are five subtypes of muscarinic receptors, M1-M5, which encode muscarinic receptor proteins that exhibit a rhodopsin-like architecture with seven transmembrane domains. ${ }^{14,15}$ Changes in the expression and/or function of muscarinic receptors have been shown to be involved in many pathophysiological processes such as degenerative nervous diseases, chronic inflammation, and cancer. ${ }^{16-18}$ In addition, muscarinic receptor activation has been shown to be involved in proliferation, angiogenesis, and tumor growth. ${ }^{19,20}$ However, the functional expression of the muscarinic type 3 receptor (M3R), a subtype that has a key role in secretory epithelia, has not yet been rigorously studied. In particular, the role of this subtype in cancer cells remains to be elucidated.

In this study, we investigated the extent to which epigenetic modulation modulates the expression of the M3R in A253 cells. These cells were originally derived from a submandibular gland carcinoma. ${ }^{21}$ In A253 cells, functional expression of the M3R has not yet been described, even though these cells originate from secretory epithelia and the $\mathrm{M} 3 \mathrm{R}$ is known to have an important role in secretion in this tissue. In contrast to HSG cells ${ }^{22}$, a $\left[\mathrm{Ca}^{2+}\right]_{\mathrm{i}}$ response to the typical muscarinic agonist is not observed in A253 cells. Thus, we hypothesized that M3R function may be suppressed on the epigenetic level, which may also contribute to the development of submandibular gland tumors. We identified a causal relationship between hypermethylation of the CpG island and the expression level of the M3R in A253 cells. We also found that demethylation at CG sites may directly activate transcription of the M3R in A253 cells, indicating that the M3R CpG island is hypermethylated.

\section{MATERIALS AND METHODS Reagents}

5-Aza-CdR was obtained from Sigma-Aldrich (St Louis, MO, USA). Antibodies against M3R were purchased from Abcam (Cambridge, UK).

\section{Cell Culture}

HSG, A253 and SGT cells originating from human salivary glands were cultured in Dulbecco's modified Eagle's medium (Welgene, Daegu, South Korea) supplemented with 10\% $(\mathrm{v} / \mathrm{v})$ fetal bovine serum (Welgene) and $1 \%$ penicillin/streptomycin (Life Technologies, Seoul, Korea). Cells were propagated at $37^{\circ} \mathrm{C}$ in a humidified atmosphere of $5 \% \mathrm{CO}_{2}$. A253 cells were pre-incubated with 5-Aza-CdR for times ranging from $4 \mathrm{~h}$ to $1 \mathrm{day}$, before the day indicated for each experiment.

\section{Human Tissue}

Human salivary gland cancer tissue was obtained from the patient with malignant neoplasm of parotid glands. After surgical excision, the tissues were immediately placed in cold $\left(4^{\circ} \mathrm{C}\right)$ physiological saline and transported to the laboratory for processing. Intact region of excised glands was used as control tissues. The use of human oral tissue specimens has followed ethical guidelines and certification approval established by the Institutional Review Board of Seoul National University Dental Hospital (CRI11023G).

\section{Reverse Transcriptase-PCR}

HSG and A253 cells were harvested for RNA extraction. Total RNA was purified using Trizol (Invitrogen, Carlsbad, CA, USA). Reverse transcriptase with an oligo-dT primer (Invitrogen) was used to prepare cDNA from $1 \mu \mathrm{g}$ of total RNA. PCR with specific primers was performed using $1 \mu \mathrm{l}$ of cDNA. The primer sets used were as follows: M1R: forward $5^{\prime}$-ACGG AGCTCCCCAAATACAG-3' ${ }^{\prime}$, reverse 5'-TAGCACATGGGG TTGATGGT-3'; M2R: forward 5'-AGCCTTCTATTTGCC AGTGA-3' ${ }^{\prime}$ reverse $5^{\prime}$-GCAACAGCACTGACTGAGGT-3'; M3R: forward 5'-GTTACCCTCATCGGACAACCT-3', reverse 5'-TTACCCACTGAGGAGTTGACG-3'; M4R: 5'-CAGTTT GTGGTGGGTAAGCG- $3^{\prime}$, reverse $5^{\prime}$-GTACAGCACCGTCA TGATGA-3'; M5R: 5'-GACCCAAGAGTCAGAAATGTG-3', reverse $5^{\prime}$-AGAAGGTAGAAACCAGGACCA-3'; GAPDH: forward $5^{\prime}$-GAAGGTGAAGGTCGGAGTC- $3^{\prime}$, reverse $5^{\prime}$-GAA GATGGTGATGGGATTTC- $3^{\prime}$. PCR conditions were as follows: 35 cycles of denaturation at $94^{\circ} \mathrm{C}$ for $30 \mathrm{~s}$, annealing at $55^{\circ} \mathrm{C}$ for $30 \mathrm{~s}$, and extension at $72{ }^{\circ} \mathrm{C}$ for $30 \mathrm{~s}$, followed by a final extension at $72{ }^{\circ} \mathrm{C}$ for $7 \mathrm{~min}$. To estimate the mRNA levels of various muscarinic receptors, cDNA from HSG cells and A253 cells was amplified for 35 cycles on a PCR thermocycler (PTC-1148C; Bio-Rad Laboratories, Hercules, CA, USA). PCR products were resolved using a Mupid-2 plus electrophoresis system (Optima, Tokyo, Japan) on 1.5\% agarose gels (Sigma-Aldrich) containing $0.1 \mu \mathrm{g} / \mathrm{ml}$ ethidium bromide (Sigma-Aldrich). PCR products were visualized under UV light with a bioimaging system (TS-312 R; Spectroline, Westbury, NY, USA).

\section{Membrane Preparation and Western Blot}

Cultured cells were collected and lysed. Cell lysates were spun by centrifugation at $600 \mathrm{~g}$ at $4{ }^{\circ} \mathrm{C}$, and the resultant supernatants were spun again at $20000 \mathrm{~g}$ at $4{ }^{\circ} \mathrm{C} .{ }^{23}$ The pellets were solubilized and then subjected to SDS/PAGE and immunoblotting. Protein concentrations were measured using the BCA protein assay kit (Pierce, IL, USA), with bovine serum albumin used as a standard. Protein samples were resolved on 8\% SDS-PAGE gels (Bio-Rad Laboratories). After electrophoretic separation using a Power-Pac Basic system (Bio-Rad Laboratories), the proteins were transferred to nitrocellulose membranes (Whatman, Dassel, Germany). The membranes were then blocked with 5\% non-fat milk (Seoul-milk, Seoul, Korea) and probed with anti-M3R antibodies (1:1500 dilution; Abcam). After washing, membranes were then incubated with HRP-conjugated secondary antibodies (Santa Cruz Biotechnology, Santa Cruz, CA, USA). Immunoreactive bands 
were ultimately visualized using ECL reagents (iNtRON Biotechnology, Sungnam, Korea).

\section{Immunofluorescence Confocal Laser Microscopy}

To visualize the M3R, HSG and A253 cells (treated with or without 5-Aza-CdR) were grown on cell culture slides. Cells were washed three times with PBS, fixed in $4 \%$ paraformaldehyde at room temperature for $15 \mathrm{~min}$, and incubated overnight at $4{ }^{\circ} \mathrm{C}$ with anti-human M3R antibodies (1:200; Abcam). Cells were washed three times with PBS containing $1 \%$ bovine serum albumin and then incubated for $1 \mathrm{~h}$ with Alexa Fluor 488 goat anti-rabbit IgG antibodies (1:200). All slides were mounted using VECTASHIELD H-1200 with DAPI (Vector Laboratories, Burlingame, CA, USA), and images were captured on a laser scanning confocal microscope (Fluoview300; Olympus, Tokyo, Japan).

\section{Measurement of $\left[\mathrm{Ca}^{2+}\right]_{i}$}

HSG and A253 cells, treated with or without 5-Aza-CdR, were loaded with the $\mathrm{Ca}^{2+}$-sensitive fluorescent probe, fura-2/AM (Molecular Probes, Eugene, OR, USA) for $45 \mathrm{~min}$ at room temperature. A MetaFluor imaging system (version 6.1; Universal Imaging, West Chester, PA, USA) was used for recording and analysis. Approximately 15-20 cells were recorded at $37^{\circ} \mathrm{C}$ and analyzed in each experiment. Fura-2 fluorescence was recorded at excitation wavelengths of 340 and $380 \mathrm{~nm}$, with an emission wavelength of $510 \mathrm{~nm}$. Results are presented as $340 \mathrm{~nm} / 380 \mathrm{~nm}$ ratios $\left(\mathrm{Ca}^{2+}\right.$ fluorescence ratio, F340/F380).

\section{Determination of 5-methylcytosine DNA Content}

Genomic DNA was extracted from HSG and A253 cells, treated with or without 5-Aza-CdR, using a Qiagen Blood and Cell Culture DNA Kit (Qiagen, Valencia, CA, USA) according to the manufacturer's recommendations. The global DNA methylation levels were then examined in the DNA samples isolated from these two salivary gland epithelial cell lines. The relative degrees of methylation in the DNA samples were quantified using a MethylFlash Methylated DNA Quantification Kit (Epigentek, Farmingdale, NY, USA) according to the manufacturer's instructions. Three replicates of each sample were performed.

\section{Methylation-specific PCR and 5-Aza-2'-Deoxycytidine Treatment}

Bisulfite modification of genomic DNA was carried out using an EpiTect Bisulfite Kit (Qiagen). Methylation-specific PCR was performed using bisulfite-treated DNA as template, using specific primer sequences for either the methylated or unmethylated form of the gene. HSG and A253 cells were seeded in 100-mm culture dishes one day before treatment. Cells were then treated with 5-Aza-CdR (Sigma-Aldrich) at a final concentration of $10 \mu \mathrm{M}$ for $1,2,3$, and 4 days. Culture medium was renewed daily, and treated cells were collected at the end of the fourth day. Genomic DNA and total RNA were then isolated using a QIAamp DNA Blood Mini Kit and an
RNeasy Mini Kit (Qiagen), respectively. PCR primers were designed using Methyl Primer Express Software, v1.0 (Applied Biosystems, Foster City, CA, USA). The following methylation-specific primers were used: $M$ forward, $5^{\prime}$-GGT TTGTGTCGATTTGATTATC- $3^{\prime}$; $M$ reverse, $5^{\prime}$-ACTCGAT ACGTAAACGACCTC- $3^{\prime}$; U forward, 5'-TTTGGTTTGT GTTGATTTGATTATT-3'; U reverse, 5' ${ }^{\prime}$-TTAACTCAATACAT AAACAACCTC-3'.

\section{Bisulfite Sequencing}

In total, $2 \mu \mathrm{g}$ of genomic DNA isolated from A253 and SGT cells, treated either with or without 5-Aza-CdR, and human tissues was modified with sodium bisulfite. The modified genomic DNA was then amplified with the following primers: forward, 5'-GGTATTTTGGTTTTGGTGATTA-3'; reverse, 5'-TCTTTCCAACAAAATATTACCAA-3'. PCR reactions were performed as follows: $94{ }^{\circ} \mathrm{C}$ for $5 \mathrm{~min}$, followed by 35 cycles of $94{ }^{\circ} \mathrm{C}$ for $40 \mathrm{~s}, 55^{\circ} \mathrm{C}$ for $40 \mathrm{~s}$, and $72{ }^{\circ} \mathrm{C}$ for $1 \mathrm{~min}$. The resultant PCR products were purified with a PCR Purification Kit (Qiagen) and ligated into pCR2.1-TOPO using the TA cloning system (Invitrogen). Plasmid-transformed TOP10 $\mathrm{F}^{\prime}$ cells were cultured overnight before isolation of plasmid DNA using a Plasmid DNA Purification Kit (iNtRON Biotechnology). Five separate clones from treated cells and five separate clones from untreated cells were chosen for sequence analysis.

\section{Statistical Analysis}

All experiments were conducted in triplicate. Statistically significant differences in the data were determined by Student's $t$-test, using GraphPad Prism 5 software (GraphPad Software, La Jolla, CA, USA). P-values less than 0.05 were considered statistically significant (designated with an asterisk; $\left.{ }^{\star} P<0.05\right)$.

\section{RESULTS}

\section{Muscarinic Receptor Subtypes in HSG and A253 Cells}

We first examined the expression levels of various muscarinic receptor subtypes in two different salivary gland epithelial cell lines, HSG and A253. Reverse transcriptase-PCR (RTPCR) was used to determine the mRNA expression levels of various muscarinic receptors. HSG cells, employed as a positive control, expressed all subtypes (1-5) of muscarinic receptors. A253 cells also expressed all subtypes of muscarinic receptors, with the exception of type 3. The M3R was expressed only at extremely low levels in A253 cells (Figure 1a), as summarized in Figure $1 \mathrm{~b}\left(n=3 ;{ }^{* *} P<0.001\right)$. These results indicated that expression of the M3R is suppressed in A253 cells. We next examined whether the low level of M3R mRNA expression is related to DNA methylation of the M3R CpG island.

\section{Induction of the M3R in A253 Cells by Treatment with 5-Aza-CdR}

We next examined whether the low expression level of the M3R is increased by 5 -Aza-CdR treatment. A time-course 
a

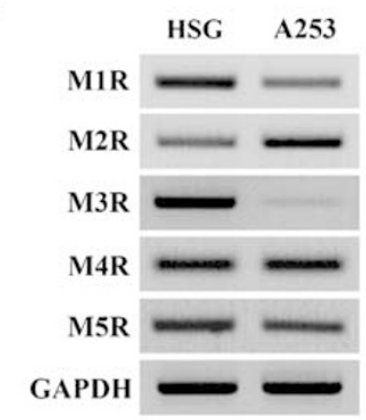

b

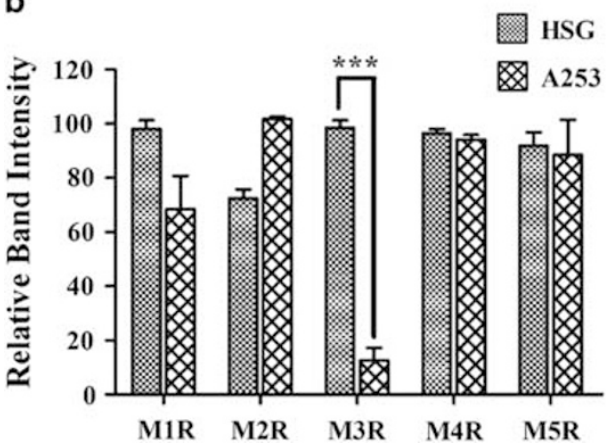

Figure 1 mRNA expression levels of various muscarinic receptor subtypes in HSG and A253 cells. (a) Total RNA was extracted from HSG and A253 cells, and reverse transcriptase-PCR (RT-PCR) was used to examine the mRNA expression levels of various muscarinic receptors. HSG cells were used as a positive control. A253 cells expressed all muscarinic receptor subtypes, except type 3 (M3R). The muscarinic receptor subtype 3 (M3R) was expressed only at an extremely low level in A253 cells. (b) Quantitative analysis of band densities. The intensity of each band was measured by densitometry, and showed that the mRNA expression level for the M3R was greatly reduced in A253 cells. The vertical bars in the graphs indicate means \pm s.d. of mRNA levels, normalized to the amount of GAPDH mRNA, as determined using RT-PCR. Experiments were repeated three times with essentially identical results; a summarized result is shown $\left(n=3 ;{ }^{* * *} P<0.001\right)$.

analysis of RT-PCR data is shown in Figure 2a. Cells were incubated with $10 \mu \mathrm{M}$ of 5 -Aza-CdR, a well-characterized demethylating agent. At $24 \mathrm{~h}$ after 5-Aza-CdR treatment, the mRNA expression level of the M3R was significantly increased. Furthermore, the expression level of M3R mRNA gradually increased in proportion to the length of the incubation period; this trend was observed even in incubation periods lasting for 4 days. After 3 days of treatment with 5-Aza-CdR, the expression level of M3R mRNA in A253 cells was nearly the same as in HSG cells, as summarized in Figure $2 \mathrm{~b}\left(n=3 ;{ }^{\star} P<0.05 ;{ }^{*} P<0.01\right)$. The increased expression level of the M3R was further confirmed at the protein level using western blot analysis (Figure 2c). Before 5-Aza-CdR treatment, only a relatively small amount of M3R protein was expressed in A253 cells. However, the amount of M3R protein increased significantly, in proportion to the length of the incubation period with 5-Aza-CdR, as summarized in Figure $2 \mathrm{~d}$

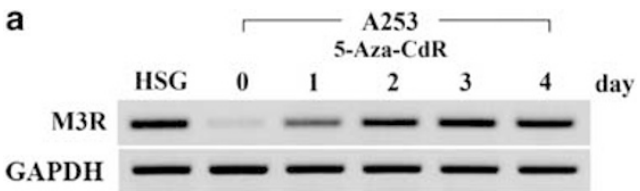

b
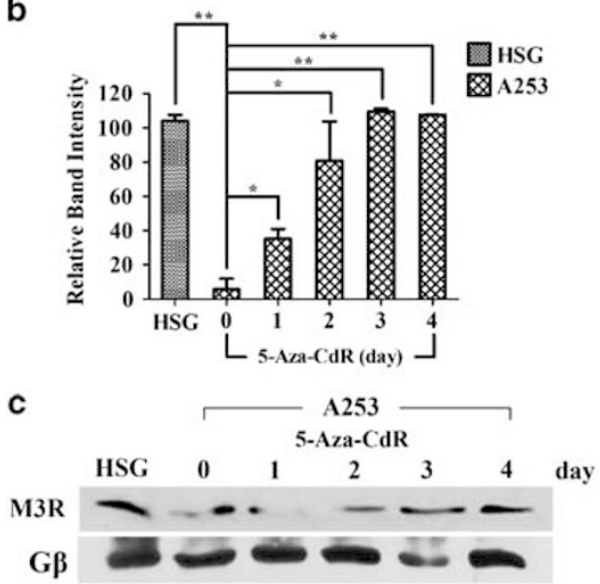

d

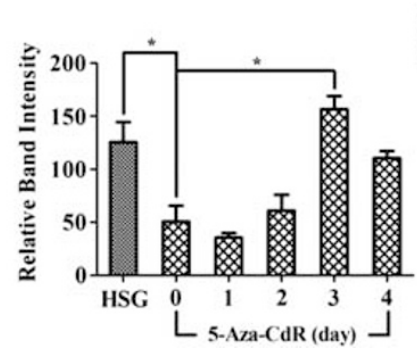

Figure 2 Induction of M3R mRNA expression and protein production by 5-aza-2'-deoxycytidine (5-Aza-CdR). A253 cells were treated with 5-Aza$\mathrm{CdR}(10 \mu \mathrm{M})$ for $24,48,72$, or $96 \mathrm{~h}$. The expression of M3R mRNA in HSG and A253 cells was then determined by reverse transcriptase-PCR $(\mathbf{a}, \mathbf{b})$ and western blot analysis (c, d). Before 5-Aza-CdR treatment, only a small amount of M3R protein was present. However, the amount of M3R protein increased proportionally with the length of the incubation period with 5-Aza-CdR. A protein with an apparent molecular weight of $66 \mathrm{kDa}$, corresponding to the M3R, was clearly observed in lysates from A253 cells after treatment with $5-A z a-C d R$ for $24-96 \mathrm{~h}$. All experiments were performed in triplicate $\left({ }^{*} P<0.05 ;{ }^{* *} P<0.01\right)$.

$\left(n=3 ;{ }^{\star} P<0.05\right)$. These results demonstrate that expression of the M3R is markedly suppressed in A253 cells and indicate that this suppression is due to hypermethylation of the M3R gene.

\section{Rescue of M3R Function by 5-Aza-CdR Treatment}

The increased level of M3R protein on 5-Aza-CdR treatment was further confirmed by confocal microscopy. Although the M3R was highly expressed in both membrane and cytosolic preparations of HSG cells (Figure 3a), the M3R was barely expressed in A253 cells (Figure 3b). However, after 3 days of treatment of A253 cells with $10 \mu \mathrm{M} 5$-Aza-CdR, the expression level of the M3R was markedly increased in both membrane and cytosolic preparations (Figure 3c). We next measured the magnitude of the intracellular free $\mathrm{Ca}^{2+}$ 
a

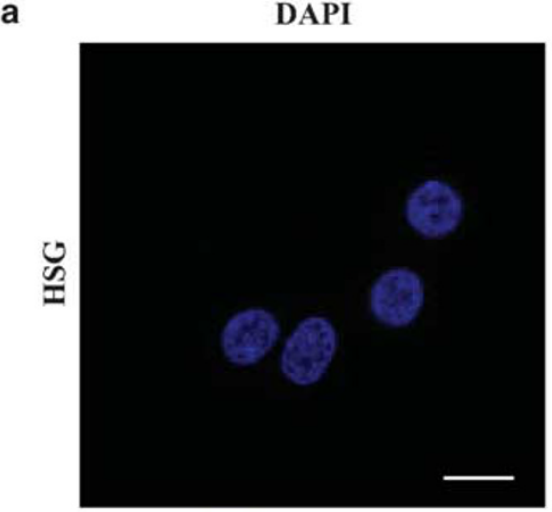

b

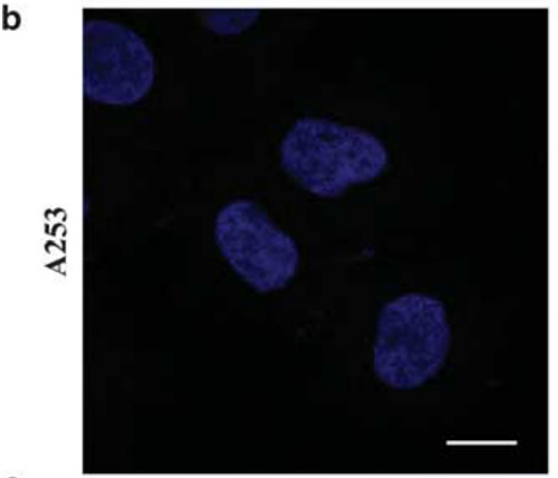

C

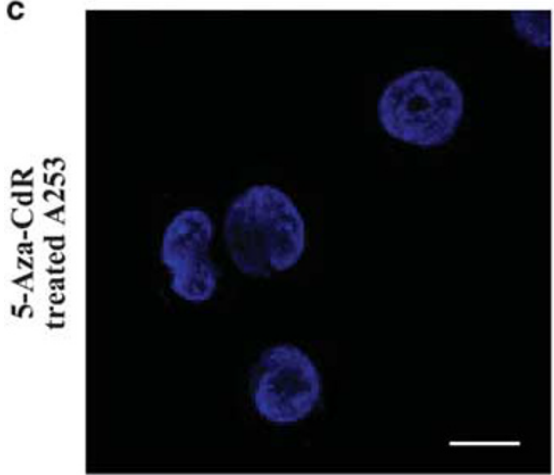

M3R
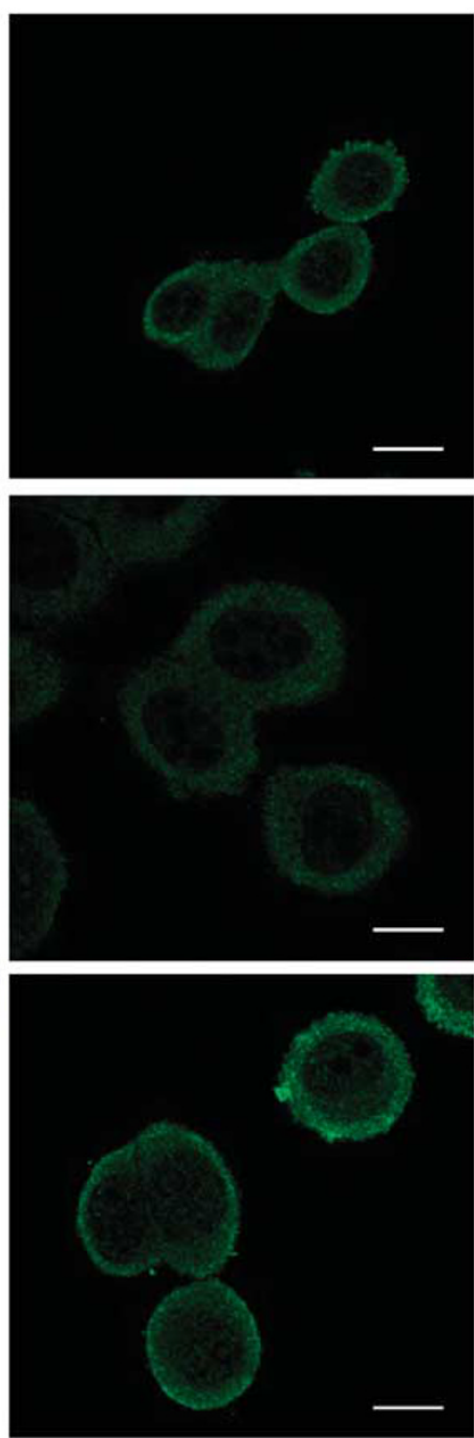

Merge
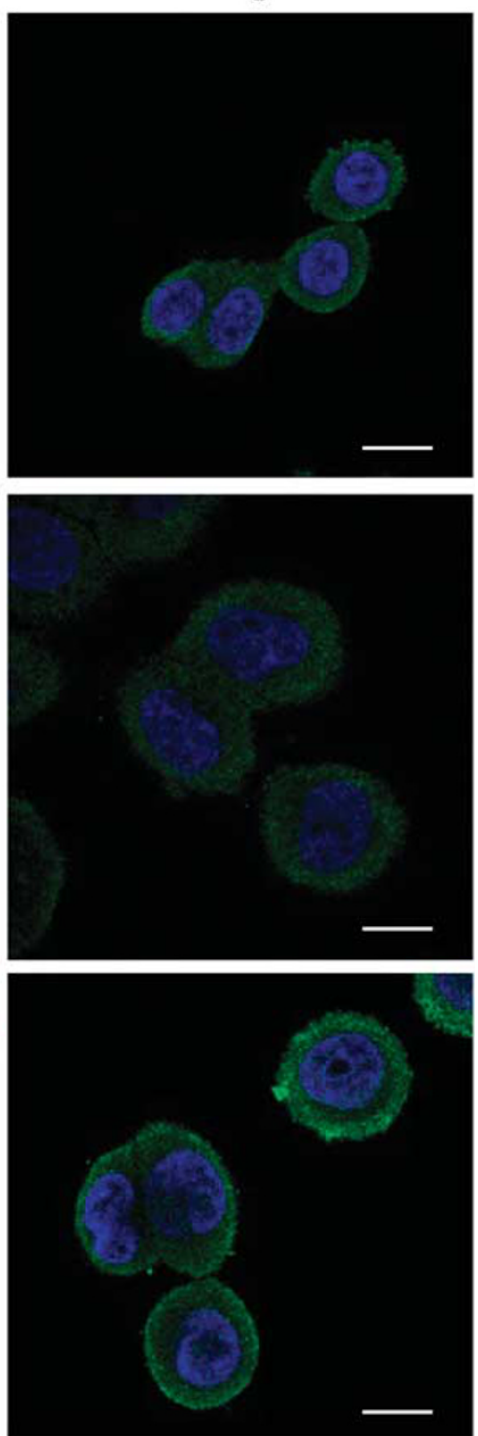

Figure 3 Immunocytochemical staining of the M3R before and after 5-aza-2'-deoxycytidine (5-Aza-CdR) treatment. The subcellular localization of the M3R was determined by immunostaining in HSG and A253 cells, both before and after 5-Aza-CdR treatment. (a) Ubiquitous expression of the M3R, both at the plasma membrane and in the cytosol, in HSG cells that were used as a positive control. (b) Expression of the M3R in A253 cells. (c) Expression of the M3R in A253 cells after 5-Aza-CdR treatment. (Original magnification, $\times 600$; scale bar, $20 \mu \mathrm{m}$ ).

$\left(\left[\mathrm{Ca}^{2+}\right]_{\mathrm{i}}\right)$ response in fura-2-loaded cells to verify that the M3R retained its function, even after demethylation. HSG cells exhibited a robust response to $10 \mu \mathrm{M}$ carbachol (CCh), a known M3R agonist, as evidenced by the increased $\left[\mathrm{Ca}^{2+}\right]_{\mathrm{i}}$ response (Figure 4a). In contrast, CCh exerted little effect on the calcium response in A253 cells (Figure 4b). Importantly, A253 cells responded well to the control stimulus ( $100 \mu \mathrm{M}$ ATP, a known purinergic agonist), as shown by the increased $\left[\mathrm{Ca}^{2+}\right]_{\mathrm{i}}$ response. After 3 days of treatment with $10 \mu \mathrm{M}$ 5 -Aza-CdR, CCh consistently evoked a calcium response in A253 cells; the amplitude of this response was not much different to that in HSG cells (Figure 4c), as summarized in Figure $4 \mathrm{~d}\left(n=3 ;{ }^{* *} P<0.001\right)$. These results demonstrate that 5-Aza-CdR treatment rescues the expression of the M3R, as well as its function.

\section{Hypermethylation of the CpG Island in A253 cells}

Our data demonstrate that hypermethylation, possibly in CpG islands, may have occurred in the M3R gene in A253 cells. Thus, we next examined the global DNA methylation levels in A253 cells, both before and after treatment with 5-Aza-CdR. We extracted genomic DNA from 5-Aza-CdRtreated and -untreated A253 cells, and then quantitatively determined the 5-methylcytosine content in the isolated genomic DNA. Untreated A253 cells exhibited significantly higher 5-methylcytosine content compared with HSG cells 

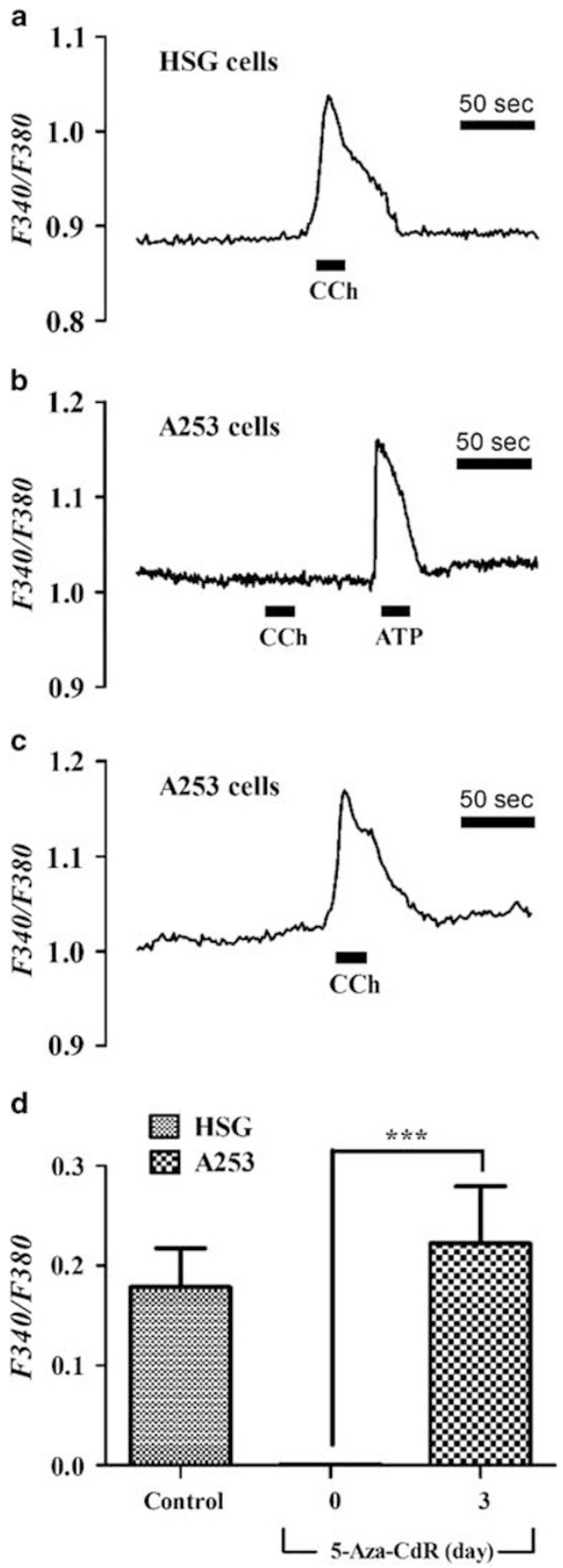

Figure 4 Rescue of M3R function by 5-aza-2'-deoxycytidine (5-Aza-CdR) treatment. Measurements of intracellular free calcium concentrations $\left(\left[\mathrm{Ca}^{2+}\right]_{\mathrm{i}}\right)$ in fura-2-loaded cells. The magnitude of the $\left[\mathrm{Ca}^{2+}\right]_{i}$ response induced by carbachol (CCh), a typical M3R agonist, was used to assess the extent of M3R function in HSG cells, untreated A253 cells, and treated A253 cells. (a) A typical $\left[\mathrm{Ca}^{2+}\right]_{i}$ response to $10 \mu \mathrm{M} \mathrm{CCh}$ in HSG cells. (b) A representative $\left[\mathrm{Ca}^{2+}\right]_{\mathrm{i}}$ response to $10 \mu \mathrm{M} \mathrm{CCh}$ and $100 \mu \mathrm{M}$ ATP in A253 cells. (c) A representative $\left[\mathrm{Ca}^{2+}\right]_{i}$ response to $10 \mu \mathrm{M} \mathrm{CCh}$ in A253 cells treated with 5-Aza-CdR. (d) Summary of all experiments, each of which was performed in triplicate $\left({ }^{* *} P<0.001\right)$. a

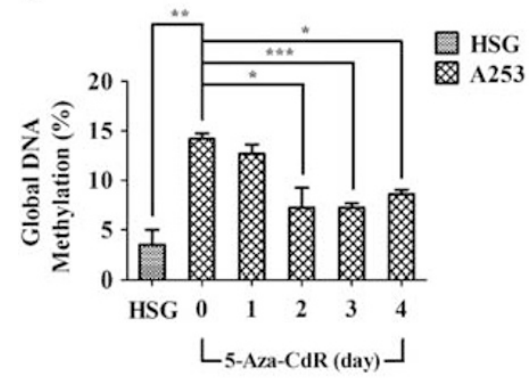

b

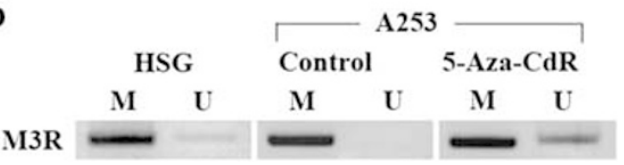

Figure 5 Methylation levels in total DNA and the M3R CpG island on treatment with 5-aza-2'-deoxycytidine (5-Aza-CdR). (a) Global DNA hypomethylation in A253 cells on 5-Aza-CdR treatment. Total DNA was extracted from HSG cells (as a control) and A253 cells, both before and after 5-Aza-CdR treatment. The 5-methylcytosine content of each preparation of DNA was then determined quantitatively. The 5-methylcytosine content decreased proportionally to the length of the incubation period with 5-Aza-CdR. (b) Induction of hypomethylation by 5-Aza-CdR in hypermethylated A253 cells. Methylation-specific PCR was performed with sodium bisulfite-modified DNA samples, obtained from either 5-Aza-CdR-treated or -untreated A253 cells. M, methylated M3R CpG island PCR products; UM, unmethylated M3R CpG island PCR products. 5-Aza-CdR treatment induced an unmethylation-specific band in A253 cells. All experiments were performed in triplicate $\left({ }^{*} P<0.05\right.$; $\left.{ }^{*} P<0.01 ;{ }^{* *} P<0.001\right)$.

(Figure 5a). However, treatment of A253 cells with 5-Aza-CdR reduced their 5-methylcytosine content; moreover, this reduction was proportional to the length of the incubation period. After 2 days of treatment with 5-Aza-CdR, the 5-methylcytosine content of A253 cells was significantly reduced to a level similar to that detected in HSG cells $\left(n=3 ;{ }^{\star} P<0.05 ;{ }^{*} P<0.01\right.$; ${ }^{* * *} P<0.001$ ). This result indicates that A253 cells (a salivary gland tumor cell line) are hypermethylated compared with HSG cells. The methylation status of the M3R CpG island was further investigated by methylation-specific PCR using DNA isolated from HSG, A253, and 5-Aza-CdR-treated A253 cells. 5-Aza-CdR treatment induced a strong unmethylation-specific band, which was not observed in untreated A253 cells (Figure 5b). This finding suggests that the expression level of the M3R is at least partially regulated by the methylation status of the M3R CpG island region.

\section{Bisulfite Sequencing of the M3R CpG Island before and After 5-Aza-CdR Treatment}

We next studied the methylation pattern within the M3R CpG island in A253, human cancer tissue, and SGT cells, before and after treatment with 5-Aza-CdR. PCR products were cloned into pCR2.1-TOPO, and five separate clones were sequenced. The DNA methylation status of each clone was determined by analyzing the sequence of the $\mathrm{CpG}$ island 
a

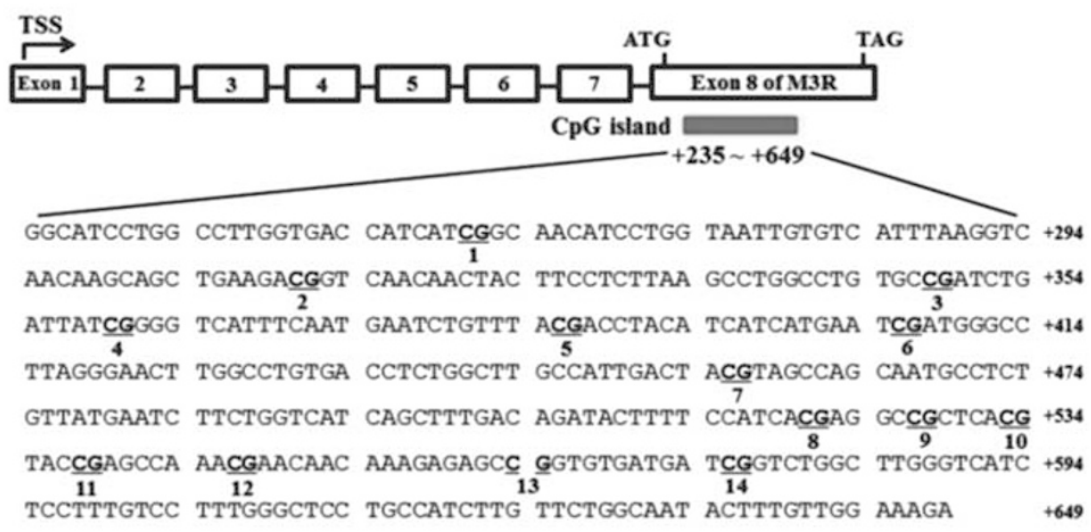

b

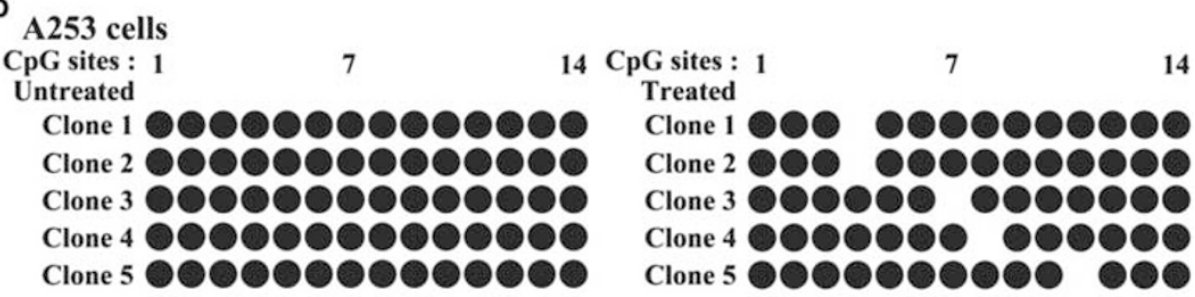

$c_{\text {human tissue }}$
CpG sites : 1
Tumor
7
14 CpG sites : 1
7
Normal
Clone 1000000000000

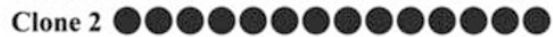
Clone 10000000000

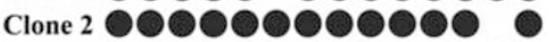

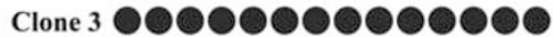
Clone 4 ९९९९९९९९९९९९९
Clone 3 ○९९
Clone 4 ००००० ○

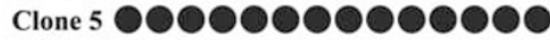
Clone 500000

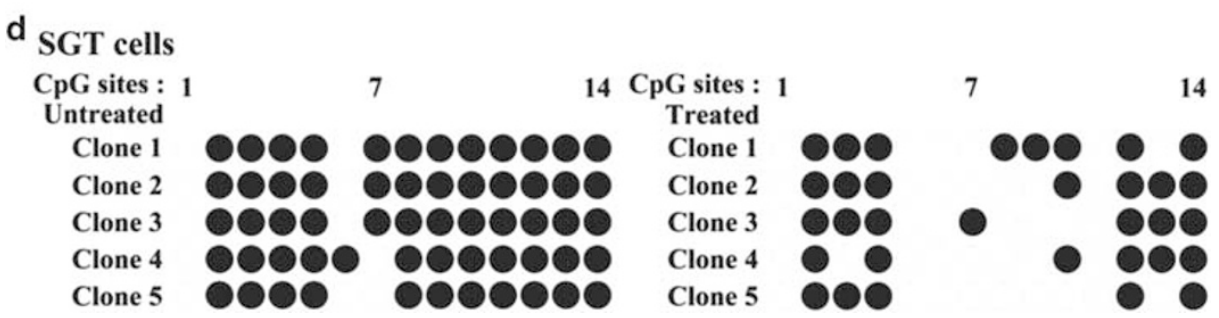

Figure 6 Bisulfite sequencing of the M3R CpG island before and after 5-aza-2'-deoxycytidine (5-Aza-CdR) treatment. (a) The M3R CpG island (GenBank no. NM_000740) was analyzed. This sequence spans from base pair +235 to +649 (relative to the start codon) and includes 14 CG pairs inside exon 8 , a coding region. The start (ATG) and stop (TAG) codons in exon 8 are shown. The CG sites within this sequence are underlined and shown in bold. TSS indicates transcription start site. (b) Bisulfite sequencing of CpG island of M3R in A253 cells. DNA isolated from A253 before (the left panel) and after 5-Aza-CdR treatment (the right panel). They were treated with bisulfite, and the M3R CpG island was PCR amplified. The resultant PCR product was ligated into PCR2.1-TOPO using the TA cloning system. Five clones before and after 5-Aza-CdR treatment were picked and sequenced. (c) Bisulfite sequencing of $\mathrm{CpG}$ island of M3R in human cancer tissue (the left panel) and normal tissue (the right panel). (d) Bisulfite sequencing of CpG island of M3R in SGT cells before (the left panel) and after 5-Aza-CdR treatment (the right panel). Symbols: $\bigcirc$, unmethylated cytosines; $\bullet$, methylated cytosines.

in the human M3R. We observed 14 CG pairs, located +235 to +649 base pairs away from the start codon of exon 8 , a coding region in the M3R gene (Figure 6a). In untreated A253 cells (the left panel in Figure 6b), all CG pairs were methylated, from the 1st to 14th CG pair. 5-Aza-CdR treatment (the right panel in Figure 6b) demethylated one of the methylated CG pairs (Figure 6b). We next examined the methylation pattern of M3R CpG island from human cancer tissue (Figure 6c). Interestingly, the result was very similar to those of A253 cells. We found that all CG pairs in M3R CpG island were also methylated (the left panel in Figure 6c). Compared with the cancer tissue, only one or two methylated CG pairs were observed in normal human tissue (the right panel in Figure 6c). Another salivary gland tumor cell line, 
SGT, showed the similar methylation pattern. Most of the CG pairs were methylated, except 1st, 6th and 7th (the left panel in Figure 6d). 5-Aza-CdR treatment also demethylated some methylated CG pairs (the right panel Figure 6d). These results indicate that $\mathrm{CpG}$ island in M3R is hypermethylated in cancer cell lines including A253 and SGT and human cancer tissues compared with normal tissues.

\section{DISCUSSION}

Epigenomic studies are well established and have been successfully applied to screen for novel candidate genes in many types of human cancer. ${ }^{24-27}$ Abnormal gene expression in tumor cells may result from alterations in copy number, sequence mutations, and/or epigenetic dysregulation. Malignant cancer cells have been shown to exhibit major disruptions in their DNA methylation profiles, and these changes have also been shown to include aberrant hypermethylation or hypomethylation of gene $\mathrm{CpG}$ islands. ${ }^{28}$

Here we employed an epigenetic strategy and compared the A253, SGT and HSG cell lines. The A253 and SGT cell lines were originally derived from a human submandibular gland tumor ${ }^{21}$, and salivary gland adenocarcinoma tissue, ${ }^{29}$ and have been used to study secretory mechanisms such as chloride transport ${ }^{30}$ and intracellular signal transduction pathways. ${ }^{31}$ Although A253 cells possess $\beta$-adrenergic and $\mathrm{P}_{2}$-purinergic receptors, they lack the functional expression of $\alpha$-adrenergic or muscarinic cholinergic receptors. ${ }^{32,33}$ Thus, in contrast to HSG cells, A253 cells do not respond to $\mathrm{CCh}$, which normally increases $\left[\mathrm{Ca}^{2+}\right]_{\mathrm{i}}$. SGT cells do not respond to CCh either (data not shown). The M3R, a GPCR, belongs to the largest transmembrane receptor superfamily of muscarinic receptors and is expressed in both humans and mice. The M3R is characterized by a seven-transmembrane $\alpha$-helix structure and is comprised of 8 exons, with the receptor-coding sequence located within exon $8 .{ }^{34,35}$ In general, the binding of a ligand to its cognate GPCR elicits $\mathrm{Ca}^{2+}$ and/or PKC signaling cascades that induce the expression of genes required for multiple fundamental functions, including exocrine and endocrine secretion, smooth muscle and cardiac muscle contraction, pain transmission, fluid homeostasis, blood pressure regulation, and immune responses. ${ }^{35}$

The M3R is well established to have a key role in fluid secretion by increasing $\left[\mathrm{Ca}^{2+}\right]_{\mathrm{i}}$. However, the expression pattern and function(s) of the M3R are still poorly understood, especially in salivary gland tumor cells such as A253 cells. We hypothesized that the mechanism of suppression of M3R function might involve hypo- and/or hypermethylation of the M3R gene in salivary gland tumor cells. In our study, 5-Aza-CdR treatment demethylated methylated CG pairs at the 4th, 7th, 8th, and 11th sites in A253 cells and at the 3rd, 5th, 8th, 9th, 10th, 11th, and 13th sites in SGT cells. Similarly, all CG pairs were methylated in cancer tissue, but 5th, 6th, 7th, 8th, 12th, and 13th CG pairs were demethylated in normal tissue. This treatment also rescued M3R function, as demonstrated by the CCh-induced calcium response observed in A253 cells. Our results are also consistent with previous studies. The CpG island of the 14-3-3 $\sigma$ gene in salivary gland adenoid cystic carcinoma cells was found to be methylated inside the exon, whereas HSG cells showed very a limited pattern of hemimethylation in the 14-3-3 $\sigma$ gene. Methylation occurring at $\mathrm{CPG}$ sites inside the exon, a coding region, has been shown to significantly reduce the expression of 14-3-3 $\sigma \cdot{ }^{36}$ Therefore, it is highly likely that internal methylation within an exon, a coding region, can affect the DNA-binding activity of transcription factors; moreover, inhibition of this methylation is likely to correlate with increased expression of the relevant gene. We found that any demethylation occurring at 14 methylated CG sites was sufficient to increase the transcriptional activity of the M3R gene, resulting in increased M3R protein expression and increased M3R function. However, it is not yet clear whether methylated CG pairs among the 14 CG sites are functionally specific or whether any site of CG demethylation is sufficient to restore M3R function.

To the best of our knowledge, this is the first study reporting that 5-Aza-CdR can epigenetically regulate the expression and function of the M3R found in salivary gland tumor cells. 5-Aza-CdR is a pyrimidine nucleoside analog that strongly inhibits DNMT activity, and is one of the strongest known inhibitors of DNA methylation. Several lines of evidence have indicated that demethylation of gene-specific sequences is associated with cell differentiation. ${ }^{37}$ At low concentrations of 5-Aza-CdR, an immortalized normal human salivary gland duct cell line lacking the expression of aquaporin 5 was shown to acquire aquaporin 5 expression and significantly increased net fluid secretion. ${ }^{38}$ Also, the 5-Aza-CdR to murine aging model C57BL/6CrSlc mice increased the volumes of salivary flow compared with those of control mice. ${ }^{39}$ In conclusion, the results of this study strongly suggest that 5-Aza-CdR-mediated demethylation in the M3R CpG island induces M3R gene expression in A253 cells, a salivary gland tumor cell line, thereby increasing the level of the M3R protein. This increased level of M3R protein restores M3R function, as evidenced by the increased $\left[\mathrm{Ca}^{2+}\right]_{i}$ response to CCh stimulation. These results suggest that hypermethylation of M3R induces the functional defect of abnormal $\left[\mathrm{Ca}^{2+}\right]_{\mathrm{i}}$ response in A253 cells compared with HSG, thus it might attenuate the secretion of saliva in patients with salivary gland tumors. Also, the loss of M3R function by hypermethylation may relate with S1P-specific GPCR because S1P1, 2, 3, and 4 receptors exist in A253 cells, whereas HSG cells show S1P1, 2, and 3 receptors only. Because S1P4 shows a limited distribution and is found only in the lymph nodes, spleen, lungs, and thymus, the expression of S1P4 in A253 cells is interesting. ${ }^{40}$ Thus, further investigation of the relationship between S1P receptors and suppressed function of M3R in salivary gland tumor may be valuable for the development of a target-specific approach for salivary gland tumor treatment. As low doses of 5-Aza-CdR have been used to treat patients with myelodysplastic syndrome and have been shown to be well tolerated by this 
group of patients ${ }^{41}$, this drug might be an effective therapeutic option for patients with salivary gland tumors.

\section{ACKNOWLEDGMENTS}

This work was supported by a grant from the national Research Foundation of Korea, through the Oromaxillofacial Dysfunction Research Center for the Elderly (No. 2013-070465) at Seoul National University in Korea.

\section{DISCLOSURE/CONFLICT OF INTEREST}

The authors declare no conflict of interest.

1. Kafri $T$, Ariel $M$, Brandeis $M$, et al. Developmental pattern of genespecific DNA methylation in the mouse embryo and germ line. Genes Dev 1992;6:705-714.

2. Laird PW, Jaenisch R. The role of DNA methylation in cancer genetic and epigenetics. Annu Rev Genet 1996;30:441-464.

3. Baylin SB, Herman JG, Graff JR, et al. Alterations in DNA methylation: a fundamental aspect of neoplasia. Adv Cancer Res 1998;72:141-196.

4. Jones PA, Laird PW. Cancer epigenetics comes of age. Nat Genet 1999:21:163-167.

5. Di Croce L, Raker VA, Corsaro M, et al. Methyltransferase recruitment and DNA hypermethylation of target promoters by an oncogenic transcription factor. Science 2002;295:1079-1082.

6. Momparler RL. Pharmacology of 5-Aza-2'-deoxycytidine (decitabine). Semin Hematol 2005;42:S9-16.

7. Egger G, Liang G, Aparicio A, et al. Epigenetics in human disease and prospects for epigenetic therapy. Nature 2004;429:457-463.

8. Cameron EE, Bachman KE, Myohanen S, et al. Synergy of demethylation and histone deacetylase inhibition in the re-expression of genes silenced in cancer. Nat Genet 1999;21:103-107.

9. Daskalakis $M$, Nguyen $\Pi$, Nguyen $C$, et al. Demethylation of a hypermethylated P15/INK4B gene in patients with myelodysplastic syndrome by 5 -Aza-2'-deoxycytidine (decitabine) treatment. Blood 2002;100:2957-2964

10. Kaneda A, Kaminishi M, Yanagihara K, et al. Identification of silencing of nine genes in human gastric cancers. Cancer Res 2002;62: 6645-6650.

11. Zhu B, Chen Z, Cheng X, et al. Induction of TRAG-3 expression in A549 lung adenocarcinoma cell line by 5 -aza-2' deoxyazacytidine. Lung Cancer 2002;38:321-322.

12. Chen H, Toyooka S, Gazdar AF, et al. Epigenetic regulation of a novel tumor suppressor gene (hDAB2IP) in prostate cancer cell lines. J Biol Chem 2003;278:3121-3130.

13. Belinsky SA, Klinge DM, Stidley CA, et al. Inhibition of DNA methylation and histone deacetylation prevents murine lung cancer. Cancer Res 2003:63:7089-7093.

14. Hulme EC, Lu ZL, Saldanha JW, et al. Structure and activation of muscarinic acetylcholine receptors. Biochem Soc Trans 2003;31:29-34.

15. Wess J, Eglen RM, Gautam D. Muscarinic acetylcholine receptors: mutant mice provide new insights for drug development. Nat Rev Drug Discov 2007;6:721-733.

16. Koch $\mathrm{HJ}$, Haas $\mathrm{S}$, Jurgens $\mathrm{T}$. On the physiological relevance of muscarinic acetylcholine receptors in Alzheimer's disease. Curr Med Chem 2005;12:2915-2921.

17. Paleari L, Grozio A, Cesario A, et al. The cholinergic system and cancer. Semin Cancer Biol 2008;18:211-217.

18. Sales ME. Muscarinic receptors as targets for anti-inflammatory therapy. Curr Opin Investig Drugs 2010;11:1239-1245.

19. Espanol AJ, Sales ME. Different muscarinc receptors are involved in the proliferation of murine mammary adenocarcinoma cell lines. Int J Mol Med 2004:13:311-317.

20. Espanol AJ, de la Torre E, Fiszman GL, et al. Role of non-neuronal cholinergic system in breast cancer progression. Life Sci 2007;80: 2281-2285.
21. Giard DJ, Aaronson SA, Todaro GJ, et al. In vitro cultivation of human tumors: establishment of cell lines derived from a series of solid tumors. J Natl Cancer Inst 1973;51:1417-1423.

22. Shirasuna K, Sato M, Miyazaki T. A neoplastic epithelial duct cell line established from an irradiated human salivary gland. Cancer 1981;48: 745-752.

23. Popova JS, Rasenick MM. Clathrin-mediated endocytosis of $\mathrm{m} 3$ muscarinic receptors. Roles for Gbetagamma and tubulin. J Biol Chem 2004;279:30410-30418.

24. Yamashita K, Upadhyay S, Osada M, et al. Pharmacologic unmasking of epigenetically silenced tumor suppressor genes in esophageal squamous cell carcinoma. Cancer Cell 2002;2:485-495.

25. Hoque MO, Kim MS, Ostrow KL, et al. Genome-wide promoter analysis uncovers portions of the cancer methylome. Cancer Res 2008;68: 2661-2670.

26. Glazer CA, Smith IM, Ochs MF, et al. Integrative discovery of epigenetically derepressed cancer testis antigens in NSCLC. PLoS One 2009:4:e8189.

27. Smith IM, Glazer CA, Mithani SK, et al. Coordinated activation of candidate proto-oncogenes and cancer testes antigens via promoter demethylation in head and neck cancer and lung cancer. PLoS One 2009;4:e4961.

28. McCabe MT, Brandes JC, Vertino PM. Cancer DNA methylation: molecular mechanisms and clinical implications. Clin Cancer Res 2009;15: 3927-3937.

29. Lee CH. Apoptotic effect of Taxol on SGT cell line. Kor J Oral Maxillofac Pathol 2008;32:139-142.

30. Roomans GM. Chloride transport in a human submandibular gland cell line studied by X-ray microanalysis. Eur J Morphol 1998;36(Suppl):132136.

31. Sugita $\mathrm{K}$, Mork AC, Zhang GH, et al. Modulation of $\mathrm{Ca}_{2}{ }^{+}$mobilization by protein kinase $C$ in the submandibular duct cell line $A 253$. Mol Cell Biochem 1999:198:39-46.

32. Marmary $\mathrm{Y}, \mathrm{He} \mathrm{XJ}$, Hand $\mathrm{AR}$, et al. Beta-adrenergic responsiveness in a human submandibular tumor cell line (A253). In Vitro Cell Dev Biol 1989;25:951-958.

33. Zhang GH, Helmke RJ, Martinez JR. Characterization of $\mathrm{Ca}_{2}^{+}$ mobilization in the human submandibular duct cell line A253. Proc Soc Exp Biol Med 1997;216:117-124.

34. Pierce KL, Premont RT, Lefkowitz RJ. Seven-transmembrane receptors. Nat Rev Mol Cell Biol 2002;3:639-650.

35. Forsythe SM, Kogut PC, McConville JF, et al. Structure and transcription of the human $\mathrm{m} 3$ muscarinic receptor gene. Am J Respir Cell Mol Biol 2002;26:298-305.

36. Uchida D, Begum NM, Almofti $A$, et al. Frequent downregulation of 14-3-3 sigma protein and hypermethylation of 14-3-3 sigma gene in salivary gland adenoid cystic carcinoma. Br J Cancer 2004;91: $1131-1138$

37. Lubbert $M$, Brugger $W$, Mertelsmann $R$, et al. Developmental regulation of myeloid gene expression and demethylation during ex vivo culture of peripheral blood progenitor cells. Blood 1996:87:447-455.

38. Motegi K, Azuma M, Tamatani $\mathrm{T}$, et al. Expression of aquaporin-5 in and fluid secretion from immortalized human salivary gland ductal cells by treatment with 5-aza-2'-deoxycytidine: a possibility for improvement of xerostomia in patients with Sjogren's syndrome. Lab Invest 2005;85:342-353.

39. Yamamura $Y$, Aota $K$, Yamanoi $T$, et al. DNA demethylating agent decitabine increases AQP5 expression and restores salivary function. J Dent Res 2012;91:612-617.

40. Seo J, Koo NY, Choi WY, et al. Sphingosine-1-phosphate signaling in human submandibular cells. J Dent Res 2010;89:1148-1153.

41. Wijermans $P$, Lubbert $M$, Verhoef $G$, et al. Low-dose 5-aza-2'deoxycytidine, a DNA hypomethylating agent, for the treatment of high-risk myelodysplastic syndrome: a multicenter phase II study in elderly patients. J Clin Oncol 2000;18:956-962. 